
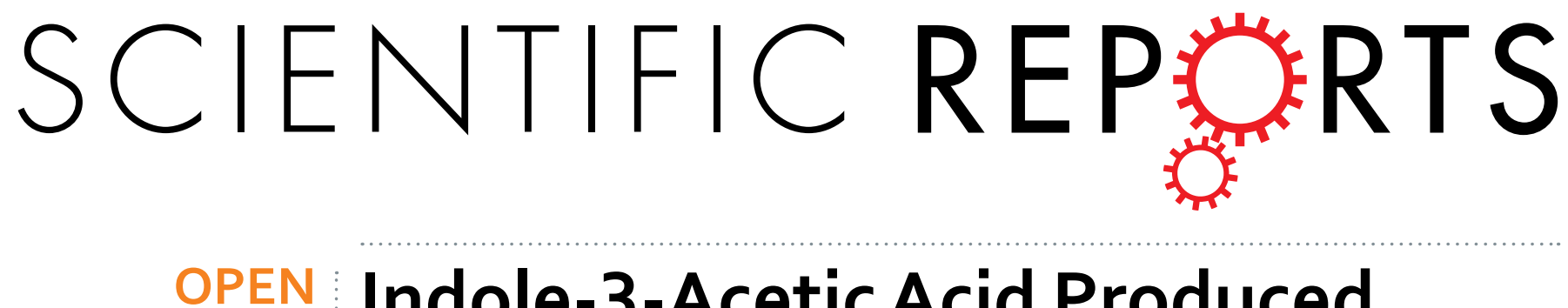

\title{
Indole-3-Acetic Acid Produced by Burkholderia heleia Acts as a Phenylacetic Acid Antagonist to \\ Disrupt Tropolone Biosynthesis in Burkholderia plantarii
}

Received: 13 October 2015

Accepted: 17 February 2016

Published: 03 March 2016
Mengcen Wang ${ }^{1,2}$, Seiji Tachibana ${ }^{1}$, Yuta Murai ${ }^{1,3}$, Li Li ${ }^{1}$, Sharon Yu Ling Lau ${ }^{1}$, Mengchao Cao ${ }^{2}$, Guonian Zhu $^{2}$, Makoto Hashimoto ${ }^{1}$ \& Yasuyuki Hashidoko ${ }^{1}$

Burkholderia heleia PAK1-2 is a potent biocontrol agent isolated from rice rhizosphere, as it prevents bacterial rice seedling blight disease caused by Burkholderia plantarii. Here, we isolated a nonantibacterial metabolite from the culture fluid of $B$. heleia PAK1-2 that was able to suppress $B$. plantarii virulence and subsequently identified as indole-3-acetic acid (IAA). IAA suppressed the production of tropolone in $B$. plantarii in a dose-dependent manner without any antibacterial and quorum quenching activity, suggesting that IAA inhibited steps of tropolone biosynthesis. Consistent with this, supplementing cultures of $B$. plantarii with either $\mathrm{L}-\left[\mathrm{ring}-{ }_{-}^{2} \mathrm{H}_{5}\right]$ phenylalanine or $\left[\right.$ ring- $\left.{ }^{2} \mathrm{H}_{2-5}\right]$ phenylacetic acid revealed that phenylacetic acid (PAA), which is the dominant metabolite during the early growth stage, is a direct precursor of tropolone. Exposure of $B$. plantarii to IAA suppressed production of both PAA and tropolone. These data particularly showed that IAA produced by $B$. heleia PAK1-2 disrupts tropolone production during bioconversion of PAA to tropolone via the ring-rearrangement on the phenyl group of the precursor to attenuate the virulence of $B$. plantarii. $B$. heleia PAK1-2 is thus likely a microbial community coordinating bacterium in rhizosphere ecosystems, which never eliminates phytopathogens but only represses production of phytotoxins or bacteriocidal substances.

Troponoids are a unique group of natural products containing a seven-membered aromatic ring with various substitutions ${ }^{1-4}$. These compounds have various bioactivities, including antiviral, antitumor, antioxidant, anti-inflammatory, and insecticidal effects ${ }^{4}$. Tropolone, which is produced by Burkholderia plantarii, is the phytotoxin responsible for rice seedling blight ${ }^{5}$. This was demonstrated when treatment only with exogenous tropolone was able to phenocopy B. plantarii infestation, which manifests as chlorosis, shoot-stunting, and root inhibition ${ }^{6,7}$.

The potent iron-chelating property of tropolone not only accounts for its broad-spectrum antimicrobial activity against bacteria and fungi ${ }^{8}$ but also contributes to its virulence and the symptoms associated with the onset of bacterial rice seedling blight ${ }^{6,9}$. When this phytotoxin accumulates in culture fluid, or is exogenously added to the culture medium, it triggers production of extracellular polysaccharide by bacteria ${ }^{10}$. Despite its key role in virulence, the tropolone biosynthetic pathway in B. plantarii remains unclear. In phytopathogenic eubacteria, production of virulence factors is often under the control of a sophisticated regulatory system ${ }^{11-14}$. In several human-pathogenic Burkholderia species, virulence factors, including capsular polysaccharide $\mathrm{I}^{15}$, helicase inhibitor $^{16}$, and actin polymerization bimABm gene ${ }^{17}$ are regulated by quorum-sensing molecules ${ }^{18-20}$.

We previously performed a screen to identify tropolone- and catechol-tolerant microbial isolates ${ }^{7}$. Here, we screened 15 of these isolates for their efficacy as biocontrol agents against $B$. plantarii. One of these isolates, Burkholderia heleia PAK1-2, suppressed blight symptoms when topically applied to rice seedlings. A

${ }^{1}$ Research Faculty of Agriculture, Hokkaido University, Kita 9, Nishi 9, Kita-ku, Sapporo 060-8589, Japan. ${ }^{2}$ Institute of Pesticide and Environmental Toxicology, Zhejiang University, No. 268 Kaixuan Road, Hangzhou 310029, China. ${ }^{3}$ Frontier Research Center for Post-Genome Science and Technology, Faculty of Advanced Life Sciences, Hokkaido University, Kita 10, Nishi 8, Kita-ku, Sapporo 060-0810, Japan. Correspondence and requests for materials should be addressed toY.H. (email: yasu-h@abs.agr.hokudai.ac.jp) 
A

B

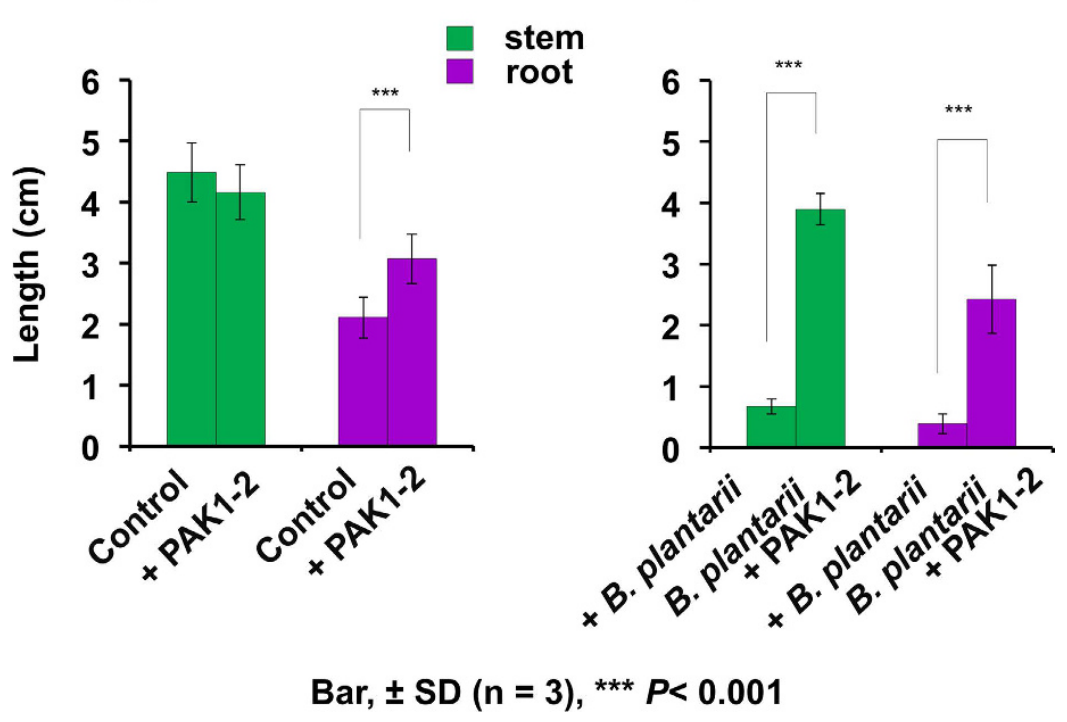

Figure 1. Biocontrol efficacy of $B$. heleia PAK1-2 against B. plantarii-caused rice seedling blight. 'Control' is the rice seedlings without any inoculation; '+PAK1-2' indicates the rice seedlings inoculated with $B$. heleia PAK1-2 only (A). Same as A, ' $+B$. plantarii' indicates rice seedlings inoculated with $B$. plantarii only, and 'B. plantarii + PAK1-2' is the rice seedlings inoculated with both B. plantarii and B. heleia PAK1-2. Values $(\mathrm{cm})$ are means \pm SD (shown by error bars) $(n=30) .{ }^{* *} P<0.001$ as determined by Student's $t$-test.

thin-layer chromatography and NMR analysis-guided bioassay that monitors tropolone production identified a non-antibacterial metabolite, indole-3-acetic acid (IAA), that was produced by B. heleia PAK1-2 and alleviated B. plantarii virulence. We show that IAA inhibits the metabolism of an intermediary compound in tropolone biosynthesis to prevent the ring re-arrangement required for the conversion of phenylacetic acid (PAA) to tropolone.

\section{Results}

Burkholderia heleia PAK1-2 is a potent biocontrol agent that blocks B. plantarii-dependent rice seedling blight. We determined that PAK1-2 was an irregular rod-shaped gram-negative bacterium of class Betaproteobacteria; alignment of its $1.5 \mathrm{kbp} 16 \mathrm{~S}$ rRNA gene sequence revealed homology with Burkholderia species (accession no. AB787501). Phylogenetic analysis for the species identification was done using multiple-aligned sequences of Burkholderia sp. PAK1-2 and other Burkholderia species including some type-strains (Figure S1). Accordingly, PAK1-2 formed a small clade together with another Burkholderia heleia strain (NBRC $\left.101817^{\mathrm{T}}\right)^{21}$ in the phylogenetic cluster of genus Burkholderia. This allowed us to positively identify PAK1-2 as a B. heleia strain.

In the dual culture system, point-inoculated B. heleia PAK1-2 showed a weak growth-inhibitory activity against $B$. plantarii (Figure S2). In addition, B. heleia PAK1-2 significantly repressed the symptoms of blight on rice seedlings infested with $B$. plantarii. The effective biocontrol properties of $B$. heleia PAK1-2 in vivo were observable both in the shoots and in the roots (Fig. 1). In particular, rice seedlings inoculated with $B$. heleia PAK1-2 had accelerated root growth and lateral root development even in the absence of $B$. plantarii infection.

Inhibition of tropolone biosynthesis by IAA produced by $B$. heleia PAK1-2. To isolate the active substance produced by $B$. heleia PAK1-2 capable of inducing $B$. plantarii cellular responses, we performed semi-quantification of tropolone production in $B$. plantarii that was exposed to fractionated metabolites of $B$. heleia PAK1-2. In contrast to untreated cells, production of tropolone by $B$. plantarii was repressed by fractions obtained by silica gel column chromatography (see Materials and Methods) at $100 \mu \mathrm{g} \mathrm{disc}^{-1}$ (Fig. $2 \mathrm{~A}$ ), and we established that this was linked to repression of cell growth (Fig. 2B,C). However, we were unable to obtain reproducible production of any growth-inhibitory compounds from large-scale cultures of B. heleia. By contrast, fraction 2 did not repress B. plantarii growth, but retained the ability to inhibit tropolone production (Fig. 2C). The active chemical compound in fraction 2 was subsequently identified as IAA (Table S1). In support of this, tropolone production was inhibited in monocultures of $B$. plantarii directly supplemented with exogenous IAA; this occurred in the absence of effects on cell growth (Fig. 2B).

Tropolone production of $B$. plantarii is under the control of the $N$-acyl-L-homoserine lactone (AHL)-QS regulatory system ${ }^{10}$. Therefore, we evaluated whether IAA-dependent repression of tropolone production was due to its modulation of the B. plantarii AHL-QS-associated genes, plaI and plaR (Figure S3). Because IAA did not elicit any statistically significant repression of both plaI and plaR transcription, it was concluded that IAA does not disrupt AHL-QS in B. plantarii.

Identification of phenyl acetic acid produced by B. plantarii at the early growth stage. B. plantarii shake-cultured for $24 \mathrm{~h}$ yielded a mixture of ethyl acetate (EtOAc)-soluble secondary metabolites, which 

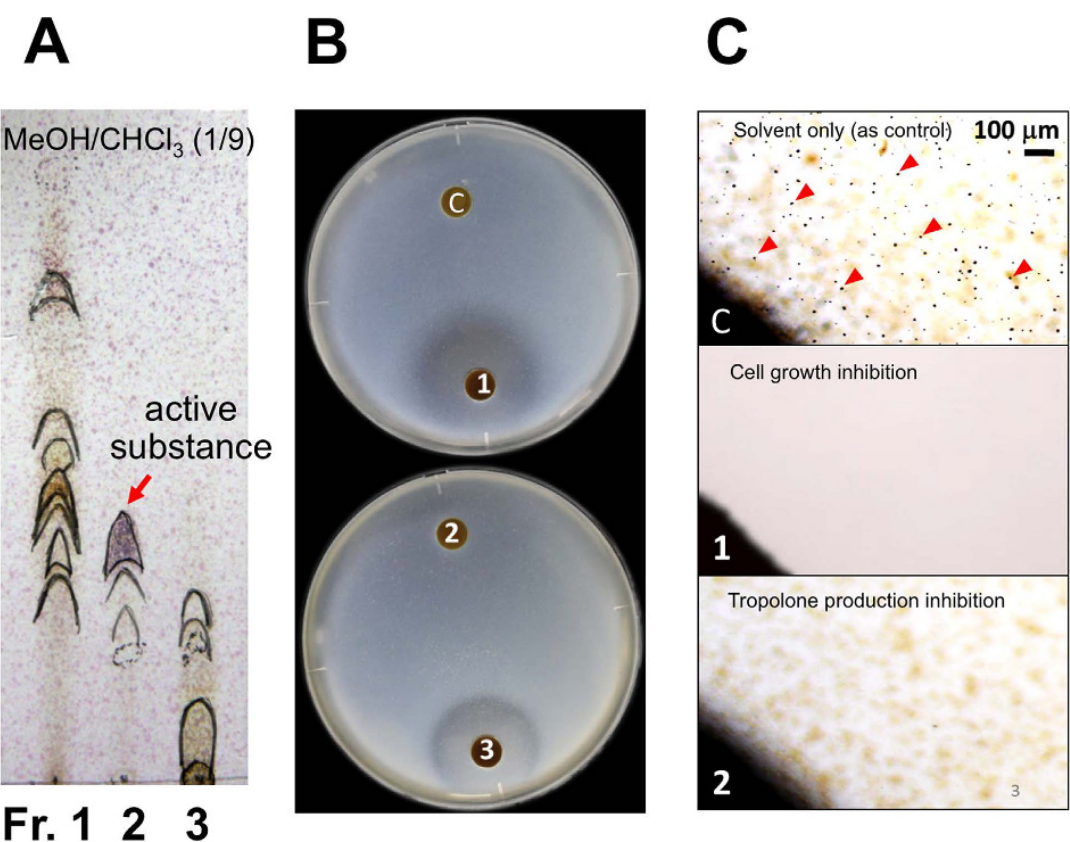

Figure 2. Tropolone production and cell growth of $B$. plantarii exposed to fractionated metabolites from B. heleia PAK1-2 or to IAA-supplemented medium. Fractions showing a similar pattern on silica gel thin-layer chromatography were combined to give three fractions $(5-20 \%, 25-50 \%$, and $60-100 \% \mathrm{MeOH} /$ chloroform for Fr. 1, 2, and 3, respectively) (A). Semi-quantitative analysis of tropolone production and $B$. plantarii growth after exposure to fractionated metabolites from B. heleia PAK1-2 loaded on paper discs (B). Microscopic observation of tropolone production by $B$. plantarii in the area around the paper disc, in which fraction $2\left(100 \mu \mathrm{g} \mathrm{disc}^{-1}\right)$ had been loaded, while cell growth and tropolone production were eliminated in the area around the paper disc loaded with fraction $3\left(100 \mu \mathrm{g} \mathrm{disc}{ }^{-1}\right)(\mathbf{C})$. Red arrow in $\mathrm{C}$ indicates the typical tropolone-iron crystals, whereas the greyish mottled objects in the background are the grown B. plantarii colonies from B. heleia-impregnated bacterial cells.

were subjected to ${ }^{1} \mathrm{H}-\mathrm{NMR}$ and MS analyses for metabolic profiling as described by Clarke and Haselden ${ }^{22}$. In direct electron impact ionization (EI)-MS, tropylium cation $(\mathrm{m} / z$ 91) appeared as the base fragment and the parent ion was predicted at $\mathrm{m} / z 136$ (Figure S4A), whereas direct field ionization (FI)-MS gave a molecular ion $\left(\mathrm{M}^{+}\right.$) peak at $m / z 136$ as the base peak (Figure S4B). In GC-MS analysis of the metabolite mixture, the most dominant peak was detected at $t \mathrm{R} 4.19 \mathrm{~min}$ in the total ion chromatographic profile (Figure S4C). For this main peak, EI for GC-MS showed a molecular ion at $m / z 136\left([\mathrm{M}]^{+}, 31 \%\right)$ and tropylium cation at $m / z 91\left([\mathrm{M}-\mathrm{COOH}]^{+}, 100 \%\right)$ (Figure S4D) consistent with phenylacetic acid (PAA) reported by Kim et al. ${ }^{23}$ (2004). In addition, the dominant compound gave the molecular ion $\left([\mathrm{M}]^{+}\right.$) peak at $\mathrm{m} / z 136$ by GC-MS under FI mode (Figure S4E). ${ }^{1} \mathrm{H}-\mathrm{NMR}$ spectrum of the EtOAc soluble mixture also showed aromatic proton and equivalent methylene proton signals, assignable to the phenyl group and the $\mathrm{C} 2$ methylene moiety of PAA (Figure S5). The most dominant metabolite produced at the early culture stage was thus identified as PAA.

Linkage of tropolone biosynthesis to accumulation of phenylacetic acid. GC-MS and NMR-based metabolic profiling showed that, before the appearance of tropolone, PAA was the major metabolite produced by $B$. plantarii during the exponential phase (Fig. 3A). Further quantitative analysis for PAA in the medium supplemented with L-phenylalanine revealed that PAA production by $B$. plantarii was sustained over $24 \mathrm{~h}$, where it reached a maximum concentration of $895.3 \mu \mathrm{M}$. However, PAA levels decreased significantly in cultures beyond $24 \mathrm{~h}$ and fell to $2 \%(13.2 \mu \mathrm{M})$ of the maximum by $72 \mathrm{~h}$. Concomitant with the rapid decrease of PAA, tropolone production accelerated robustly beginning at $24 \mathrm{~h}$ and reached its maximum level $(763.9 \mu \mathrm{M})$ at $60 \mathrm{~h}$ (Fig. 3A). Tropolone production dose-dependently increased when $B$. plantarii was fed with 0.5-2 mM PAA (Fig. 3B). Together, these qualitative and quantitative data reveal a close link between upstream PAA production and downstream tropolone production, and strongly suggest that PAA is the direct precursor of tropolone.

PAA can be derived from shikimic acid or via an oxidative decarboxylation-coupled deamination of L-phenylalanine. Therefore, conversion of L-phenylalanine to tropolone via PAA by B. plantarii was also confirmed by supplementing medium containing the phytopathogen with L-phenylalanine instead of PAA (Fig. 3C). However, PAA induced greater tropolone production than that observed with L-phenylalanine at the same concentration $(2 \mathrm{mM})$.

After growth in PD broth media supplemented with L-phenylalanine- $\left[\right.$ ring $\left.-{ }^{2} \mathrm{H}_{5}\right]$ for $36 \mathrm{~h}$, the PAA produced was extracted from the acidified culture fluid. PAA- $\left[\right.$ ring- $\left.-{ }^{2} \mathrm{H}_{5}\right]$ from labelled L-phenylalanine and non-deuterated PAA from L-phenylalanine or shikimate originally in the medium or biosynthesized by $B$. plantarii from ingredients in the plain medium were present in a ratio of 3.6:1 (calculated from the relative peak intensity in FI-MS 
A

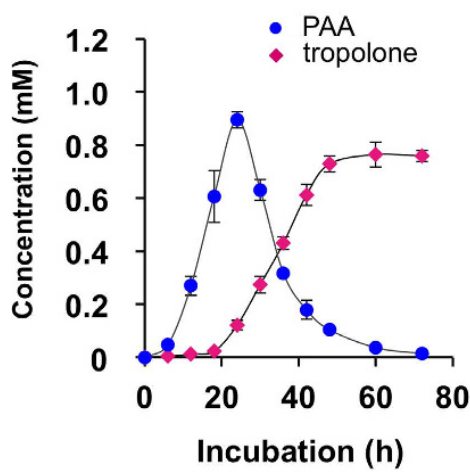

B

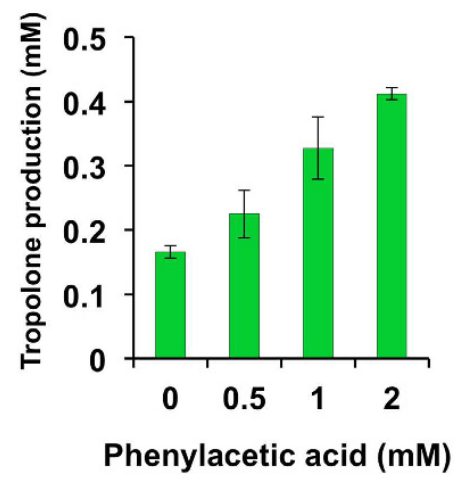

C

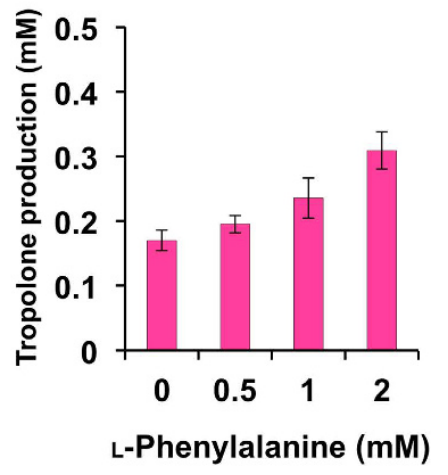

Figure 3. Relationship between tropolone production and dose of phenylacetic acid or L-phenylalanine in B. plantarii. Kinetics of tropolone and PAA production in B. plantarii $(\mathbf{A})$. Dose-dependent promotion of tropolone production in $B$. plantarii by addition of exogenous PAA at a concentration range of $0.5-2 \mathrm{mM}$ (B). Dose-dependent promotion of tropolone production in B. plantarii by addition of L-phenylalanine at a concentration range of $0.5-2 \mathrm{mM}(\mathbf{C})$. Values are means $\pm \mathrm{SD}$ (shown by error bars) $(n=3)$.

spectrum) (Fig. 4). The incorporation rate of $\mathrm{L}-\left[\right.$ ring- $\left.^{2} \mathrm{H}_{5}\right]$ phenylalanine into PAA was $78.3 \%$. Further incorporation of deuterium atoms of $\left[\right.$ ring- $\left.^{2} \mathrm{H}_{5}\right] \mathrm{PAA}$ into tropolone led to tropolone- $d_{4}$ and tropolone- $d_{3}$ with a ratio of 1.6:1. These data are consistent with PAA being the direct precursor of tropolone.

Treatment of PAA with TfOD yielded a mixture of deuterated PAA with 2- and 5-substituted deuterium atoms on the benzene ring. GC-MS/MS analysis revealed that a mixture of $\left[\right.$ ring- $\left.{ }^{2} \mathrm{H}_{2-}\right]$ PAA was converted by $B$. plantarii to tropolone- $d_{2 \sim 4}$ (Figure S6). This experiment provided evidence that PAA is the direct precursor of tropolone in $B$. plantarii. The absence of one deuterium on the labelled tropolone molecule may indicate that the benzene ring is rearranged concomitantly with an aromatic proton exchange.

Interference of the tropolone biosynthetic pathway of $B$. plantarii by indole-3-acetic acid and its analogous compounds. In the presence of $500 \mu \mathrm{M}$ IAA, PAA levels decreased from $980 \mu \mathrm{M}$ to $240 \mu \mathrm{M}$, representing $76 \%$ repression of PAA production $\left(\mathrm{LC}_{50}=c a .125 \mu \mathrm{M}\right)($ Fig. $5 \mathrm{~A})$. IAA likely prevents the metabolism of L-phenylalanine, but it is also likely that the suppression of decarboxylation-coupled deamination on L-phenylalanine by IAA is not the main mode of action in the inhibition of tropolone production by $B$. plantarii. Conversely, $500 \mu \mathrm{M}$ IAA prevented $92 \%$ of tropolone production $\left(\mathrm{LC}_{50}=c a .90 \mu \mathrm{M}\right)$, mainly from PAA, showing that the repressive effect of IAA against tropolone production is mediated through inhibition of the rearrangement of the benzene ring of PAA into a seven-membered tropolone ring (Fig. 5B).

Neither indole nor a series of the other indolic analogues tested suppressed PAA production by $B$. plantarii cells, whereas tropolone production was slightly repressed upon exposure to the indole derivatives, except for IAA. IAA reduced tropolone levels by two fold. Cell growth of $B$. plantarii was unaffected by the indole derivatives, including IAA (Figure S7).

Interference of the tropolone biosynthetic pathway of $B$. plantarii by PAA analogues. In the paper disk assay using PD agar medium impregnated with $B$. plantarii cells, PAA analogues were loaded on a paper disk as $10 \mu \mathrm{L}$ of each $1 \mu \mathrm{M}$ solution or the respective diluted solutions. Among the PAA analogues tested, $p$-tolylacetic acid, $(R)-(-)$-2-methylphenylpropionic acid, and ( $p$-isopropylphenyl)acetic acid inhibited tropolone production as effectively as IAA at the same concentration (Fig. 6).

\section{Discussion}

IAA is a well-known pro-growth plant phytohormone that induces cell division and lateral root development ${ }^{24}$. Here, we show that IAA is a non-antibacterial diffusible component that blocks tropolone synthesis and suppresses rice seedling blight disease. This finding reveals that IAA is not simply a hormone but actually a key mediator between plant and tropolone-producing B. plantarii to regulate virulence of the phytopathogen. This may be the mechanism by which indole suppresses shigatoxin production in Escherichia coli ${ }^{25}$.

Based on a report on tropone biosynthesis in the marine bacterium Phaeobacter gallaeciensis, class Alphaproteobacteria ${ }^{26}$, we investigated the mechanism by which IAA interferes with tropolone production. PAA is an aromatic metabolite that is produced when B. plantarii are fed L-phenylalanine, and it accumulates in the culture fluid ${ }^{27}$. PAA production by $B$. plantarii is thus directly linked to tropolone production, and levels of PAA were particularly elevated in PD broth cultures in the present study (Fig. 5). The main carbon source in PD broth medium is glucose, whereas the nitrogen source is a mixture of amino acids primarily composed of non-aromatic L-leucine, L-aspartic acid, L-glutamic acid, and L-glutamine; only a minor amount of aromatic amino acids, i.e., $\mathrm{L}$-phenylalanine and L-tyrosine, is present ${ }^{28,29}$. We also confirmed production of PAA by $B$. plantarii in minimum salt medium ${ }^{9}$ (data not shown). Therefore, we attribute the high productivity of PAA in B. plantarii to an active shikimate pathway ${ }^{30}$ along with decarboxylation-coupled deamination of exogenous L-phenylalanine ${ }^{31}$, suggesting that PAA is the direct precursor of tropolone. 

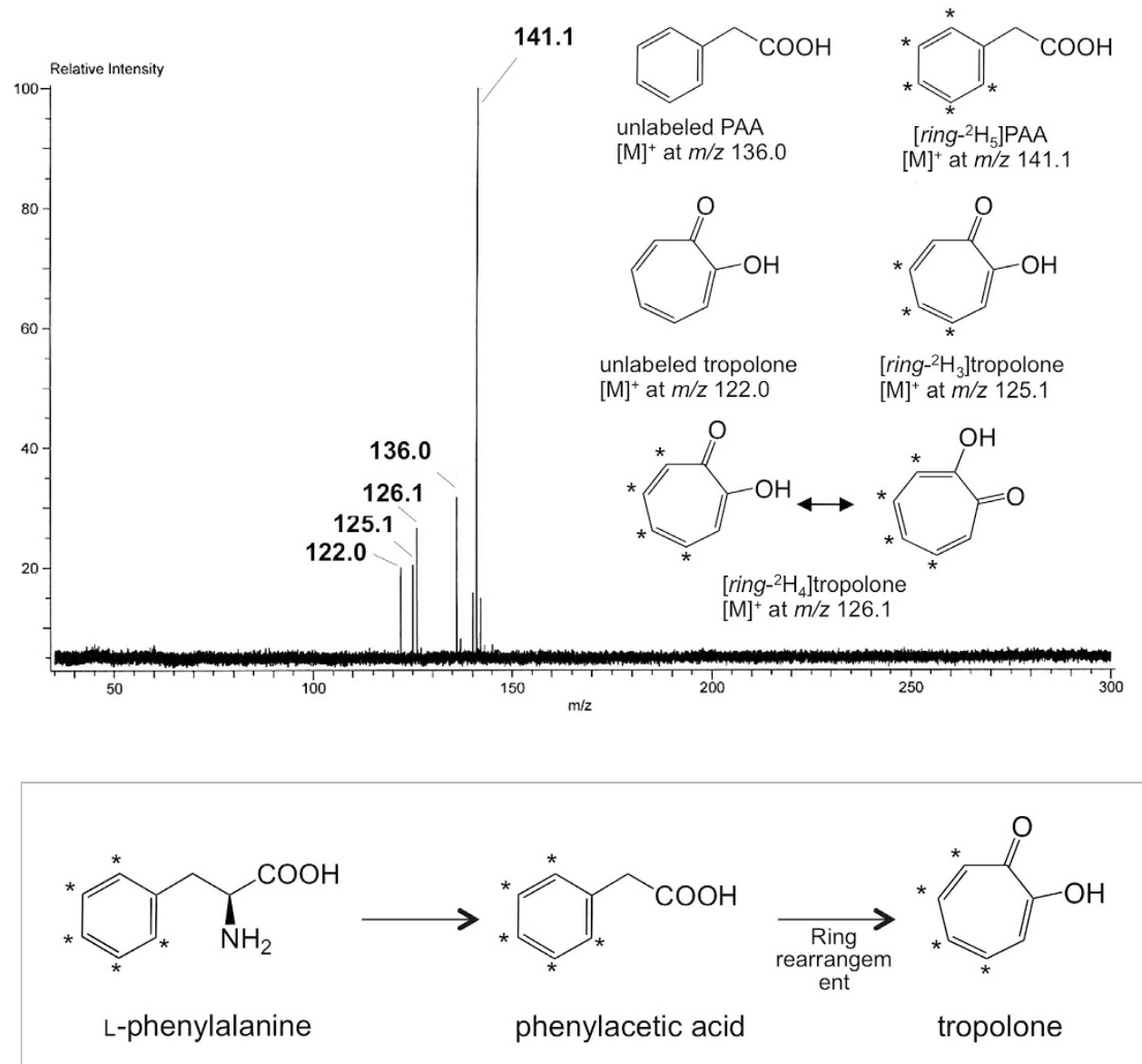

Figure 4. Identification of isotopomeric tropolones and phenylacetic acids from L- $\left[\right.$ ring $\left.^{2}{ }^{2} \mathrm{H}_{5}\right]$ phenylalanine. FI-MS spectrum of metabolites corresponding to PAA and tropolone obtained from $B$. plantarii supplemented with L- $\left[\right.$ ring- $\left.{ }^{2} \mathrm{H}_{5}\right]$ phenylalanine as shown in the top panel. The structures of tropolone, PAA and their isotopomers deduced from the molecular weights are also shown in the same panel. In the conversion of $\mathrm{L}$ - $\left[\right.$ ring- $\left.{ }^{2} \mathrm{H}_{5}\right]$ phenylalanine into $\left[\right.$ ring- $\left.{ }^{2} \mathrm{H}_{4}\right]$ tropolone via $\left[\right.$ ring $\left.-{ }^{2} \mathrm{H}_{5}\right] \mathrm{PAA}$, one deuterium at the C-2 or C-6 position was replaced with a proton during the ring rearrangement (bottom panel). Asterisk indicates the position of the deuterated carbon.

A

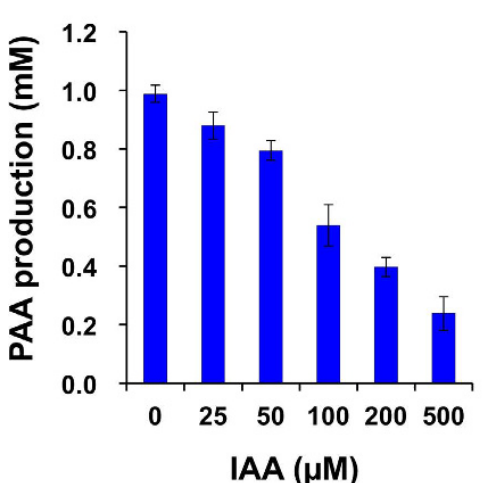

B

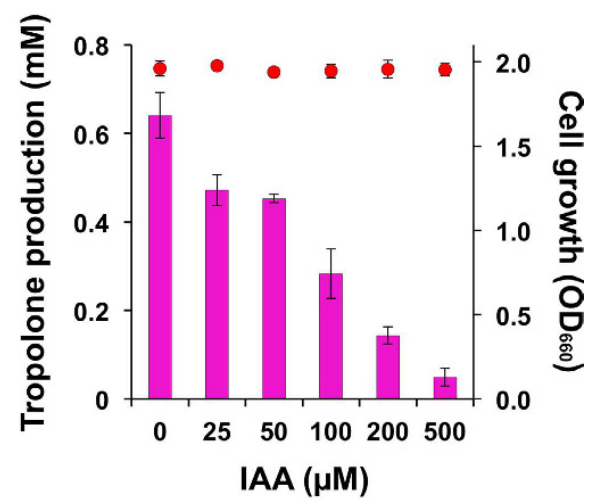

Figure 5. Effect of indole-3-acetic acid on PAA and tropolone production by $B$. plantarii. Production of both PAA (A) and tropolone (B) was quantified from B. plantarii PD broth cultures containing $25-500 \mu \mathrm{M}$ IAA. Values are the mean $\pm \mathrm{SD}$ (shown as error bars) $(n=3)$. The cell growth of each shake culture was also monitored based on the optical density at $660 \mathrm{~nm}$ (shown by red circles in panel B). 
<smiles>O=C(O)Cc1cccnc1</smiles>

3-pyridylacetic acid $\cdot \mathrm{HCl}($ A)<smiles>CC(C)c1ccc(CC(=O)O)cc1</smiles>

(p-isopropylphenyl)acetic acid (E)<smiles>O=C(O)Cc1ccc(O)cc1</smiles>

4-hydroxyphenylacetic acid (B)<smiles>C[C@H](C(=O)O)c1ccccc1</smiles>

(R)-(-)-2-methylphenylpropionic acid $(\mathbf{F})$<smiles>O=C(O)Cn1ccnc1</smiles>

1-imidazoleacetic acid (C)<smiles>CCC(C(=O)O)c1ccccc1</smiles>

(士)-2-phenylbutyric acid (G)<smiles>Cc1ccc(CC(=O)O)cc1</smiles>

p-tolylacetic acid (D)<smiles>O=C(O)[C@H](O)Cc1ccccc1</smiles>

(S)-(-)-3-phenyllactic acid (H)<smiles>O=C(O)Cc1c[nH]c2ccccc12</smiles>

\begin{tabular}{|c|c|c|c|c|c|c|c|c|c|}
\hline $\begin{array}{c}\text { Compound } \\
\text { Amount loaded }\end{array}$ & A & B & C & D & E & F & G & H & I \\
\hline $\mathbf{1}(1$ umol $)$ & - & - & - & + & ++ & ++ & \pm & - & ++ \\
\hline $\mathbf{2}(100 \mathrm{nmol})$ & - & - & - & - & \pm & - & - & - & - \\
\hline $\mathbf{3}(10 \mathrm{nmol})$ & - & - & - & - & $\mathrm{nt}$ & $\mathrm{nt}$ & - & - & - \\
\hline $\mathbf{4}(1 \mathrm{nmol})$ & - & - & - & - & $\mathrm{nt}$ & $\mathrm{nt}$ & - & - & - \\
\hline
\end{tabular}

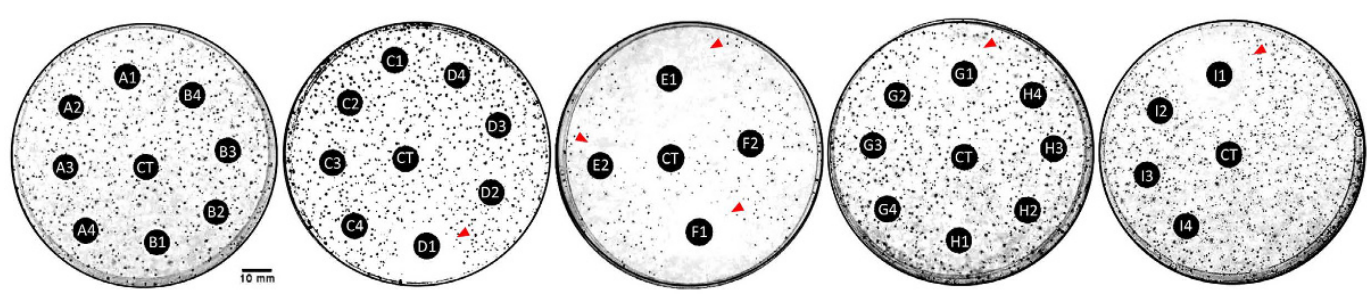

Figure 6. Effect of compounds structurally similar to PAA on production of tropolone by B. plantarii. Eight compounds, i.e., 3-pyridylacetic acid $\mathrm{HCl}$ (A), 4-hydroxyphenylacetic acid (B), 1-imidazoleacetic acid (C), p-tolylacetic acid (D), ( $p$-isopropylphenyl)acetic acid (E), (R)-(-)-2-methylphenylpropionic acid (F), $( \pm)$-2-phenylbutyric acid $(\mathbf{G})$, and $(S)-(-)$-3-phenyllactic acid $(\mathbf{H})$, along with indole-3-acetic acid (IAA) (I) as positive control, were tested on $B$. plantarii-impregnated gellan plates, in which Winogradsky's mineral mixture supplemented with $50 \mathrm{~g} \mathrm{~L}^{-1}$ sucrose, $500 \mathrm{mg} \mathrm{L}^{-1}$ yeast extract, and $0.1 \mathrm{mM} \mathrm{Fe}_{2}\left(\mathrm{SO}_{4}\right)_{3}$ was solidified with $10 \mathrm{~g} \mathrm{~L}^{-1}$ gellan gum. An $\mathrm{MeOH}$ solution of each test compound prepared at $0.1,1,10$, and $100 \mathrm{mM}$ was loaded on a paper disk for the assay, and the absolute amount of the test compound on each paper disc is shown in a sub-table in the panel. The sub-number shows the absolute amount of the compound loaded (e.g. A2 is 3-pyridylacetic acid $\cdot \mathrm{HCl}$ at $100 \mathrm{nmol})$. CT is the control ( $\mathrm{MeOH}$ only). Red arrows on the plate photograph indicate tropolone inhibition zone.

Feeding experiments using deuterium-labelled L-phenylalanine showed that L-phenylalanine is effectively converted to not only deuterium-labelled PAA but also deuterium-labelled tropolone (Fig. 4). Furthermore, direct feeding of deuterium-labelled PAA to $B$. plantarii led to incorporation of deuterium on the phenyl group into the tropolone seven-membered ring (Figure S6). Thus, tropolone biosynthesis in B. plantarii involves bioconversion of PAA to tropolone via an aromatic ring-rearrangement. This process is similar to the steps in the biosynthetic pathway of a tropolone-type metabolite, tropodithietic acid, in P. gallaeciensis (e.g., transamination and oxidative decarboxylation of phenylalanine to PAA ${ }^{26}$. PAA production in P. gallaeciensis was not observed when it was cultured in medium containing no L-phenylalanine, suggesting that L-phenylalanine is the most important precursor of PAA in P. gallaeciensis ${ }^{30}$. The reason why four but not five carbons were labelled in tropolone converted from $\left[\right.$ ring- $\left.{ }^{2} \mathrm{H}_{5}\right]$-PAA remains unclear; however, the mechanisms of ring rearrangement should be investigated in future studies by using ${ }^{13} \mathrm{C}$-labeled PAA at the $\mathrm{C} 2$-position.

Exposure of $B$. plantarii to IAA during the exponential phase of culture growth suppressed PAA production, whereas IAA more effectively decreased PAA-derived tropolone production (Fig. 5). IAA is structurally analogous to PAA and acts as a competitive inhibitor mainly against ring rearrangement on PAA for conversion into tropolone. IAA produced by the potent antagonistic bacterium $B$. heleia against bacterial rice blight disease thus demonstrated disturbance of virulence-associated phytotoxin production by the bacterial pathogen without any antibacterial mode of action. These data suggest that IAA-mediated biological and chemical control strategies against virulence of $B$. plantarii via tropolone production could attenuate symptom development and restore growth and extension of the roots. Similarly, IAA is known as the mediator of attenuation of potato tuber lesions by Fusarium solani f. sp. eumartii, where activity of the chymotrypsin-like Fusarium extracellular serine protease was shown to be dose-dependently inhibited by exogenous IAA without any fungal growth repression ${ }^{32}$.

In summary, the insight that we have provided into microbial interspecies interaction in the rhizosphere ecosystem may lead to the development of novel biocontrol agents. Such agents may reduce the symptoms caused by phytopathogens while allowing them to survive and contribute to a well-balanced rhizosphere ecosystem. 


\begin{abstract}
Methods
Analytical instruments and chemicals. Primary analytical instruments and chemicals used were as follows: Waters 600 HPLC (Waters, MA, USA) with an L-column2 ODS column $(250 \mathrm{~mm} \times$ i.d. $4.6 \mathrm{~mm}$; particle size $5 \mu \mathrm{m}$ ); Agilent 218 Purification Systems (Agilent, Santa Clara, CA, USA) with a Prep-C18 column $(50 \mathrm{~mm} \times$ i.d. $30 \mathrm{~mm}$; particle size $5 \mu \mathrm{m})$; Agilent 7890A GC (Agilent, CA, USA) with an HP-5 capillary column $(30 \mathrm{~m} \times$ i.d. $0.25 \mathrm{~mm}$ ); MS spectrometers JEOL JMS-T100GCV, JMS-SX-102 (JEOL, Tokyo, Japan) and Agilent 7000C GC-MS/MS (Santa Clara, CA, USA) with an Agilent HP-5 glass capillary column (Agilent Technologies, $30 \mathrm{~m} \times$ i.d. $0.32 \mathrm{~mm}$ ) under the conditions of initial temperature of $100^{\circ} \mathrm{C}$ for $1.5 \mathrm{~min}$ and heating at a rate of $60^{\circ} \mathrm{C}$ min $^{-1}$ until $300^{\circ} \mathrm{C}$ with a carrier gas of He; NMR spectrometers JEOL JNM-EX270 (JEOL, Tokyo, Japan) and Bruker AM 500 (Bruker, Bremen, Germany); ABI Prism 310 Genetic Analyzer (Applied Biosystems, CA, USA); StepOnePlus Real-Time PCR thermal cycling block (Applied Biosystems, CA, USA); trifluoromethanesulfonic acid- $d$ (TfOD, Energy Chemical, Shanghai, China); L-phenylalanine (TCI, Tokyo, Japan); L-phenylalanine[ring- ${ }^{2} \mathrm{H}_{5}$ ] (Sigma-Aldrich, MO, USA); phenylacetic acid (PAA, Sigma-Aldrich, St. Luis, MO, USA). Other chemicals used for preliminary screenings in the present study were purchased from TCI and Wako (Osaka, Japan).
\end{abstract}

Bacterial strains and culture conditions. B. plantarii was kindly provided by Professor Yuichi Takikawa (Faculty of Agriculture, Shizuoka University) and Kumiai Chemical Industry Co. Ltd. (Tokyo, Japan). Thirteen bacterial species previously screened from the rice rhizosphere as tolerable to $10 \mathrm{mM}$ catechol $^{7}$ were tested for biocontrol efficacy against rice seedling blight. All of the bacterial species were routinely grown at $25^{\circ} \mathrm{C}$ in the dark in potato dextrose (PD) broth ( $\mathrm{pH}$ 6.2) either statically or with agitation at $110 \mathrm{rpm}$. Alternatively, cultures were grown on a PD agar plate that was solidified with $1.5 \%$ agar (Wako, Osaka, Japan).

Biocontrol test against bacterial rice seedling blight. Seeds of rice (Oryza sativa var. japonica cv. Koshihikari) surface-sterilized with $0.5 \%$ hypochlorite and $70 \%$ ethanol were subjected to inoculation with $B$. plantarii by means of soaking in $10 \mathrm{~mL}$ of $B$. plantarii cell suspension $\left(10^{3}\right.$ colony forming units $\left.(\mathrm{CFU}) \mathrm{mL}^{-1}\right)$ in Petri dishes. For treatment with test bacteria, the seeds were simultaneously inoculated with $50 \mu \mathrm{L}$ of the bacterial cell suspension $\left(10^{6} \mathrm{CFU} \mathrm{mL} \mathrm{mL}^{-1}\right)$ and antagonistic efficacy was evaluated. Surface-sterilized rice seeds infested with B. plantarii only (control) or the testing bacterium only were also prepared along with those that were not inoculated with bacteria. All of the rice seeds were incubated, transplanted, and subsequently cultivated as previously described $^{10}$. After $5 \mathrm{~d}$ of cultivation, the length of shoots and roots and the appearance or abrogation of symptoms such as discoloration of the shoot were measured.

Metabolic changes of $\boldsymbol{B}$. plantarii following co-culture with $\boldsymbol{B}$. heleia PAK1-2. B. heleia PAK1-2 was point-inoculated at the centre of a PD agar plate impregnated with $B$. plantarii cells as previously described ${ }^{10}$. After 3-d incubation, colony morphology was observed under a light microscope (Olympus IX70, Tokyo, Japan). Plain PD agar plates point-inoculated with $B$. plantarii only or B. heleia only were used as controls. PD broth medium inoculated with $B$. plantarii and $B$. heleia PAK1-2 (both at $10^{3} \mathrm{CFU} \mathrm{mL} \mathrm{mL}^{-1}$ ) or B. plantarii only (for control) was quantitatively monitored for tropolone production. After $72-\mathrm{h}$-shake-culturing, the culture fluid was subjected to solid-phase extraction and HPLC.

Tropolone production assay using $B$. plantarii-cell-impregnated agar plates. PD-agar medium containing $0.1 \mathrm{mM} \mathrm{FeSO}_{4}$ and viable cells of $B$. plantarii were prepared for bioassay to identify tropolone production inhibitors as follows: PD broth medium supplemented with $1.0 \%$ agar was autoclaved at $120^{\circ} \mathrm{C}$ for $20 \mathrm{~min}$ and cooled to below $50^{\circ} \mathrm{C}$; then, $1 \mathrm{~mL}$ of $10 \mathrm{mM} \mathrm{FeSO}_{4}$ solution filtered through a sterile membrane $(0.4 \mu \mathrm{m}$ cellulose acetate, Millipore, Darmstadt, Germany) was added and mixed well. The liquefied PD agar mixed well with the cell suspension of pre-cultured Burkholderia plantarii $\left(10 \mathrm{~mL}\right.$ of bacterial cell suspension as $\left.10^{6} \mathrm{CFU} \mathrm{mL}^{-1}\right)$ was then cast on plastic Petri plates (Bio-Bik, Ina-Optika Co. Ltd, Osaka, Japan). This agar medium impregnated with $B$. plantarii cells was used in the screening assay for inhibitors against production of tropolone. For a more sensitive assay, Winogradsky's medium-based gellan plate $\left(50 \mathrm{~g} \mathrm{~L}^{-1}\right.$ sucrose, $0.5 \mathrm{~g} \mathrm{~L}^{-1}$ yeast extract, $10 \mathrm{~g} \mathrm{~L}^{-1}$ gellan gum) containing $0.1 \mathrm{mM} \mathrm{FeSO}_{4}$ was used instead.

A paper disk with a diameter of $9 \mathrm{~mm}$ and thickness of $0.5 \mathrm{~mm}$, on which a solution of a test compound was loaded, was placed on a $9-\mathrm{cm}$ sterile Petri plate containing $10 \mathrm{~mL}$ of medium. In the test, paper disks that had absorbed $10 \mu \mathrm{L}$ of a test compound solution at each concentration and then been air-dried were placed on the assay plates followed by incubation at $28^{\circ} \mathrm{C}$ for 5 days. When the test compounds showed inhibitory activity against tropolone production observed as tiny but visible particles of dark reddish precipitated crystals, the assay plate around the paper disc was visible as a turbid background area without any dark crystalline particles of tropolone because $B$. plantarii cell growth was permitted.

Metabolic profiling of $B$. plantarii using ${ }^{1} \mathrm{H}-\mathrm{NMR}, \mathrm{GC}$ and mass spectroscopy. B. plantarii was inoculated into $50 \mathrm{~mL}$ of PD broth medium $\left(10^{3} \mathrm{CFU} \mathrm{mL}{ }^{-1}\right)$ and shake-cultured for $24 \mathrm{~h}$ at $25^{\circ} \mathrm{C}$. After removal of bacterial cells, the culture fluid was adjusted to $\mathrm{pH} 3.5$ with $2 \mathrm{M}$ sulphuric acid and extracted twice with $50 \mathrm{~mL}$ of EtOAc. The organic layer was dried over anhydrous $\mathrm{Na}_{2} \mathrm{SO}_{4}$, concentrated, and re-dissolved in EtOAc for metabolic profiling according to the method described by Clarke and Haselden ${ }^{22}$.

A half portion of the EtOAc solute was concentrated and re-dissolved in methanol- $d_{4}$ for ${ }^{1} \mathrm{H}-\mathrm{NMR}$ analysis without any purification process. The other half of the EtOAc solute was further concentrated to $100 \mu \mathrm{L}$, of which $0.2 \mu \mathrm{L}$ was subjected to GC-MS analysis using Agilent 7890A GC in combination with JMS-SX-102 MS spectrometer to obtain a GC profile of total ion chromatography. The EI fragmentation pattern of the most dominant chemical component was recorded by the GC-MS analysis. In parallel, the concentrated sample of the crude mixture was directly applied for FI-MS and EI-MS analyses. 
Isolation and identification of the B. heleia PAK1-2-derived compound IAA that represses tropolone production in $B$. plantarii. To collect secondary metabolites produced by $B$. heleia PAK1-2, $3 \mathrm{~mL}$ of $B$. heleia PAK1-2 cell suspension $\left(10^{6} \mathrm{CFU} \mathrm{mL}^{-1}\right)$ was inoculated into a 3-L culture of PDB and shake-cultured for $3 \mathrm{~d}$ at $25^{\circ} \mathrm{C}$ in the dark. Bacterial cells were separated by centrifugation at $8500 \times \mathrm{g}$ for $10 \mathrm{~min}$. The culture fluid was passed through Cosmosil 75C18-OPN (250 g, Nacalai Tesque, Kyoto, Japan) on a Buchner funnel previously conditioned with $\mathrm{MeOH}(2 \mathrm{~L}$ ) followed by an excess of Milli-Q water (3 L). After removal of the void water by vacuum, the substances trapped by the Cosmosil particles were eluted with a sufficient volume of $\mathrm{MeOH}$ $(2 \mathrm{~L})$. The eluates $(318 \mathrm{mg})$ were concentrated and suspended in chloroform; then, they were subjected to silica gel column chromatography ( $50 \mathrm{~g}, \mathrm{GF}_{60} 35$ to 70 mesh, Merck, Darmstadt, Germany) by stepwise elution with $1 \%$ to $100 \% \mathrm{MeOH}$ in chloroform to obtain 10 fractions. Fractions exhibiting similar thin-layer chromatography profiles were combined to obtain three main fractions (fractions 1, 2, and 3 for $5-20 \%, 25-50 \%$, and $60-100 \%$ $\mathrm{MeOH} /$ chloroform respectively). To search for active principles that repressed tropolone production, these fractionated metabolites were subjected to an agar diffusion assay on B. plantarii-impregnated PD agar containing $0.1 \mathrm{mM} \mathrm{FeCl}_{3}{ }^{10}$. Physicochemical properties of the active principle isolated from fraction 2 are shown in Table S1.

Identification and quantification of phenyl acetic acid produced by $B$. plantarii. Phenylacetic acid (PAA) was identified as the main metabolite of $B$. plantarii in the early growth stage (Fig. 2). Cultures of B. plantarii in PD broth medium were sampled at $0,6,12,18,24,30,36,42,48,60$ and $72 \mathrm{~h}$ in a time-course experiment. Each culture was sampled $(2 \mathrm{~mL})$ in triplicate and centrifuged at $8500 \times g$ for $10 \mathrm{~min}$, and then the resulting supernatant $(1.5 \mathrm{~mL})$ was then subjected to solid phase extraction with a preconditioned C18 cartridge of $200 \mathrm{mg}$ resin (Sep-Pak Vac 3cc, Waters, Milford, MA, USA). The resulting cartridge column was washed with water $(3 \mathrm{~mL})$, the water was voided, and then the column was eluted with methanol $(3 \mathrm{~mL})$. The methanolic elutes were concentrated and re-dissolved in $150 \mu \mathrm{L}$ of methanol, of which $10 \mu \mathrm{L}$ was injected into an HPLC system with an isocratic mobile phase of MeOH:10 mM phosphoric acid (85:15) and a photodiode array detector (at $260 \mathrm{~nm}$ ). The retention time $(t R)$ of PAA was $3.9 \mathrm{~min}$. Standard solutions $(10 \mu \mathrm{L})$ of PAA $(0.01,0.1,0.5,1$ and $10 \mathrm{mM})$ were also subjected to HPLC for preparation of a PAA standard curve. This yielded an equation of $y=0.0001 x-0.216$ $\left(R^{2}=0.996\right) . y=$ concentration of PAA $(\mathrm{mM}), x=$ absolute peak intensity of PAA.

A conventional feeding experiment for tropolone production was performed for B. plantarii using PD broth media supplemented with L-phenylalanine at $0.5,1$, and $2 \mathrm{mM}$ in triplicate at each concentration, and PD broth media containing exogenous PAA at $0.5,1$, and $2 \mathrm{mM}$ were run in triplicate. After $36 \mathrm{~h}$ of shake-culture, each culture medium was also subjected to HPLC analysis for quantification of tropolone production.

Deuterium-labelled precursors and feeding experiments. Additional feeding experiments were performed using deuterium-labelled substrate candidates. B. plantarii was shake-cultured in $50 \mathrm{~mL}$ of PD broth medium supplemented with $2 \mathrm{mM}$ commercially available L-phenylalanine- $\left[\right.$ ring $\left._{-}{ }^{2} \mathrm{H}_{5}\right]$. EtOAc extracts from the 36-h-shake-cultured fluid were subjected to FI-MS analysis (JEOL JMS-T100GCV) without further purification.

Deuterium-labelled PAA was obtained by treatment of authentic PAA $(500 \mathrm{mg})$ with TfOD $(6.5 \mathrm{~mL}, 20$ equivalents) at room temperature for $12 \mathrm{~h}$ according to a previously reported approach ${ }^{33}$. The reaction mixture was gently diluted in $50 \mathrm{~mL}$ of Milli-Q water and subjected to liquid-liquid partition with chloroform ( $50 \mathrm{~mL}$, twice). The organic layer was dried with anhydrous $\mathrm{Na}_{2} \mathrm{SO}_{4}$, concentrated, re-dissolved in $\mathrm{MeOH}$ and purified by Agilent 218 preparative HPLC to yield PAA- $\left[\right.$ ring $\left.^{2} \mathrm{H}_{2}{ }^{-}\right](419 \mathrm{mg})$ (Fig. 4). The mixture of deuterated PAA was further added to PD broth media at $\sim 1.4 \mathrm{mM}\left(200 \mathrm{mg} \mathrm{L}^{-1}\right)$ for shake-culture of $B$. plantarii. The culture fluid after $36 \mathrm{~h}$ of incubation was extracted with EtOAc; concentrated substances were re-dissolved in EtOAc $(100 \mu \mathrm{L})$ and subjected to FI-MS and GC-MS/MS (in $1 \mu \mathrm{L}$ portions) for detection of deuterated tropolone and qualitative analysis for the deuteration ratio.

Gene expression analysis using quantitative real time-PCR for plal and plaR genes associated with the AHLs-OS system. To investigate the mechanism by which tropolone production is repressed by IAA, we examined the expression of QS-associated genes, particularly those related with AHL. To this end, B. plantarii was shake-cultured for $12 \mathrm{~h}$ in plain PD broth medium (control) or PD broth that contained IAA $(200 \mu \mathrm{M})$ and examined for expression of plaI (AHLs synthase gene) and plaR (AHLs receptor gene) using real-time quantitative reverse transcription PCR (qRT-PCR). Isolation of total RNA, digestion of the remaining genomic DNA, cDNA synthesis, and removal of remaining RNA were sequentially conducted as reported previously ${ }^{10}$, and qRT-PCR was performed in a StepOnePlus Real-Time PCR thermal cycling block (Applied Biosystems, CA, USA). Specific-primers for the qRT-PCR were plaI RT-forward (5'-GGA AGA CGA AAA ATT CGA G-3')/plaI RT-reverse (5'-TAC ACC GGT ATC GTC G-3'); plaR RT-forward (5'-GAG ATC AAC AGC CTG AC- $\left.3^{\prime}\right) /$ plaR RT-reverse ( $5^{\prime}$-AGC GAA TGC GAG AGA T-3'); and rpoD RT-forward (5'-CTA CAA GTC GAA GTC CTA C- $\left.3^{\prime}\right) / r p o D ~ R T$-reverse ( $5^{\prime}$-ATC GAC ATC AGT TCG TTC- $3^{\prime}$ ). An initial step of $30 \mathrm{~s}$ at $95^{\circ} \mathrm{C}$ followed by 30 cycles of $5 \mathrm{~s}$ at $95^{\circ} \mathrm{C}, 30 \mathrm{~s}$ at $52^{\circ} \mathrm{C}$, and $1 \mathrm{~min}$ at $72^{\circ} \mathrm{C}$ w performed. Fold changes in the expression of each target gene was calculated in comparison to those in the internal control gene rpoD (RNA polymerase sigma factor RpoD) according to the $2^{-\Delta \Delta C T}$ method $^{34}$.

Statistical analyses. Statistical analysis was performed using Microsoft Excel 2012 for Student's $t$ and Student-Newman-Keuls tests with appropriate calculation of statistically significant differences as indicated. $P<0.05$ was considered significant.

\section{References}

1. Dewar, M. J. S. Structure of stipitatic acid. Nature 155, 50-51, doi: 10.1021/ja01146a101 (1945).

2. Nozoe, T. Substitution products of tropolone and allied compounds. Nature 167, 1055-1057, doi: 10.1038/1671055a0 (1951)

3. Doering, W. V. E. \& Knox, L. H. Tropolone. J Am Chem Soc 73, 828-838 (1951). 
4. Zhao, J. Plant troponoids: Chemistry, biological activity and biosynthesis. Curr Med Chem 14, 2597-2621, doi: 10.2174/ 092986707782023253 (2007).

5. Azegami, K. et al. Tropolone as a root growth-inhibitor produced by a plant pathogenic Pseudomonas sp. causing seedling blight of rice. Ann Phytopathol Soc Jpn 51, 315-317, doi.org/10.3186/jjphytopath.51.315 (1985).

6. Azegami, K. et al. Pseudomonas plantarii sp. nov, the casual agent of rice seedling blight. Int J Syst Bacteriol 37, 144-152. doi: 10.1099/00207713-37-4-475 (1987)

7. Wang, M., Hashimoto, M. \& Hashidoko, Y. Carot-4-en-9,10-diol, a conidiation-inducing sesquiterpene diol produced by Trichoderma virens PS1-7 upon exposure to chemical stress from highly active iron chelators. Appl Environ Microbiol 79, 1906-1914, doi: 10.1128/aem.03531-12 (2013).

8. Wakimoto, S. et al. Production of antibiotics by plant pathogenic pseudomonads. Ann Phytopathol Soc Jpn 52, 835-842, doi: 10.3186/jjphytopath.52.835 (1986).

9. Azegami, K., Nishiyama, K. \& Kato, H. Effect of iron limitation on "Pseudomonas plantarii" growth and tropolone and protein production. Appl Environ Microbiol 54, 844-847, doi: 10.1016/s0168-1605(03)00316-7 (1988).

10. Wang, M., Hashimoto, M. \& Hashidoko, Y. Repression of tropolone production and induction of a Burkholderia plantarii pseudobiofilm by carot-4-en-9,10-diol, a cell-to-cell signaling disrupter produced by Trichoderma virens. Plos One 8, e78024, doi: 10.1371/ journal.pone.0078024 (2013).

11. Fuqua, C. \& Greenberg, E. P. Listening in on bacteria: acyl-homoserine lactone signalling. Nat Rev Mol Cell Biol 3, 685-695, doi: $10.1038 / \mathrm{nrm} 907$ (2002).

12. von Bodman, S. B., Bauer, W. D. \& Coplin, D. L. Quorum sensing in plant-pathogenic bacteria. Ann Rev Phytopathol 41, 455-482, doi: 10.1146/annurev.phyto.41.052002.095652 (2003).

13. Barnard, A. M. L. et al. Quorum sensing, virulence and secondary metabolite production in plant soft-rotting bacteria. Philos Trans R Soc B-Biol Sci 362, 1165-1183, doi: 10.1098/rstb.2007.2042 (2007).

14. Cataldi, T. R., Bianco, G., Fonseca, J. \& Schmitt-Kopplin, P. Perceiving the chemical language of Gram-negative bacteria: listening by high-resolution mass spectrometry. Anal Bioanal Chem 405, 493-507, doi: 10.1007/s00216-012-6371-2 (2013).

15. Reckseidler, S. L., DeShazer, D., Sokol, P. A. \& Woods, D. E. Detection of bacterial virulence genes by subtractive hybridization: identification of capsular polysaccharide of Burkholderia pseudomallei as a major virulence determinant. Infect Immun 69, 34-44, doi: 10.1128/iai.69.1.34-44.2001 (2001).

16. Cruz-Migoni, A. et al. A Burkholderia pseudomallei toxin inhibits helicase activity of translation factor eIF4A. Science 334, 821-824, doi: 10.1126/science.1211915 (2011).

17. Sarovich, D. S. et al. Variable virulence factors in Burkholderia pseudomallei (melioidosis) associated with human disease. PLoS ONE 9, e91682, doi: 10.1371/journal.pone.0091682 (2014).

18. Venturi, V. et al. Quorum sensing in the Burkholderia cepacia complex. Res Microbiol 155, 238-244, doi: 10.1016/j. resmic.2004.01.006 (2004).

19. Deng, Y., Boon, C., Eberl, L. \& Zhang, L.-H. Differential modulation of Burkholderia cenocepacia virulence and energy metabolism by the quorum-sensing signal BDSF and its synthase. J Bacteriol 191, 7270-7278, doi: 10.1128/jb.00681-09 (2009).

20. Schmid, N. et al. The AHL- and BDSF-dependent quorum sensing systems control specific and overlapping sets of genes in Burkholderia cenocepacia H111. PLoS ONE 7, e49966, doi: 10.1371/journal.pone.0049966 (2012).

21. Aizawa, T., Ve, N. B., Nakajima, M. \& Sunairi, M. Burkholderia heleia sp. nov., a nitrogen-fixing bacterium isolated from an aquatic plant, Eleocharis dulcis, that grows in highly acidic swamps in actual acid sulfate soil areas of Vietnam. Int J Syst Evol Micr 60, 1152-1157, doi: 10.1099/ijs.0.015198-0 (2010).

22. Clarke, C. J. \& Haselden, J. N. Metabolic profiling as a tool for understanding mechanisms of toxicity. Toxicol Pathol 36, 140-147, doi: $10.1177 / 0192623307310947$ (2008).

23. Kim, Y. et al. Identification and antimicrobial activity of phenylacetic acid produced by Bacillus licheniformis isolated from fermented soybean, chungkook-jang. Curr Microbiol 48, 312-317, doi: 10.1007/s00284-003-4193-3 (2004).

24. Teale, W. D., Paponov, I. A. \& Palme, K. Auxin in action: signalling, transport and the control of plant growth and development. Nat Rev Mol Cell Bio 7, 847-859, doi: 10.1038/nrm2020 (2006).

25. Bommarius, B. et al. A family of indoles regulate virulence and shiga toxin production in pathogenic E. coli. PLoS ONE 8, e54456, doi: 10.1371/journal.pone.0054456 (2013).

26. Thiel, V. et al. Identification and biosynthesis of tropone derivatives and sulfur volatiles produced by bacteria of the marine Roseobacter clade. Org Biomol Chem 8, 234-246, doi: 10.1039/b909133e (2010).

27. Teufel, R. et al. Bacterial phenylalanine and phenylacetate catabolic pathway revealed. Proc Nal Acad Sci 107, 14390-14395, doi: 10.1073/pnas.1005399107 (2010).

28. Hughes, B. P. The amino-acid composition of potato protein and of cooked potato. Brit J Nutr 12, 188-195, doi: 10.1079/ bjn19580026 (1958).

29. Ohara-Takada, A. et al. Change in content of sugars and free amino acids in potato tubers under short-term storage at low temperature and the effect on acrylamide level after frying. Biosci Biotechnol Biochem 69, 1232-1238, doi: 10.1271/bbb.69.1232 (2005).

30. Berger, M. et al. Genetic analysis of the upper phenylacetate catabolic pathway in the production of tropodithietic acid by Phaeobacter gallaeciensis. Appl Environ Microbiol 78, 3539-3551, doi: 10.1128/AEM.07657-11 (2012).

31. Stafford, H. A. \& Lewis, L. L. Conversion of L-phenylalanine and D-phenylalanine to phenylacetate via phenylpyruvate in sorghum leaf extracts. Plant Physiol 64, 176-181, doi: 10.1104/pp.64.2.176 (1979).

32. Terrile, M. C., Olivieri, F. P., Bottini, R. \& Casalongué, C. A. Indole-3-acetic acid attenuates the fungal lesions in infected potato tubers. Physiol Plantarum 127, 205-211, doi: 10.1111/j.1399-3054.2006.00667.x (2006).

33. Murai, Y. et al. Rapid and controllable hydrogen/deuterium exchange on aromatic rings of $\alpha$-amino acids and peptides. Eur J Org Chem 2013, 5111-5116, doi: 10.1002/ejoc.201300405 (2013).

34. Livak, K. J. \& Schmittgen, T. D. Analysis of relative gene expression data using real-time quantitative PCR and the $2^{\mathrm{T} \Delta \Delta \mathrm{C}}$ method. Methods 25, 402-408, doi: 10.1006/meth.2001.1262 (2001).

\section{Acknowledgements}

We thank Dr. E. Fukushi and Mr Y. Takada (GC-MS and NMR Laboratory, Research Faculty of Agriculture, Hokkaido University) for their assistance in MS and NMR analyses. We also thank Y. Takikawa (Shizuoka University) and Kumiai Chemical Industry Co. Ltd. for providing us B. plantarii. This work was supported by the commission for Development of Artificial Gene Synthesis Technology for Creating Innovative Biomaterial from METI, Japan (to Yasuyuki Hashidoko) and a Grant-in-aid for Scientific Research A and B from the Japan Society for the Promotion of Science (no. 20240833 and 26304042 to Yasuyuki Hashidoko). This work was also supported by NSFC (31501684), SRF for ROCS, SEM (J20141150), and Fundamental Research Funds for the Central Universities (2015QNA6015) to Mengcen Wang and Guonian Zhu. 


\section{Author Contributions}

Y.H. and M.W. designed the experiments; M.W., S.T., Y.M., M.C., L.L. and S.Y.L.L. conducted the experiments; M.W., Y.H. and M.H. collected, assembled and analysed data; MW drafted the article and Y.H. did critical revision of the article for intellectual content; Y.H., M.W. and G.Z. received financial supports; All authors reviewed the manuscript.

\section{Additional Information}

Supplementary information accompanies this paper at http://www.nature.com/srep

Competing financial interests: The authors declare no competing financial interests.

How to cite this article: Wang, M. et al. Indole-3-Acetic Acid Produced by Burkholderia heleia Acts as a Phenylacetic Acid Antagonist to Disrupt Tropolone Biosynthesis in Burkholderia plantarii. Sci. Rep. 6, 22596; doi: 10.1038/srep22596 (2016).

(c) (i) This work is licensed under a Creative Commons Attribution 4.0 International License. The images or other third party material in this article are included in the article's Creative Commons license, unless indicated otherwise in the credit line; if the material is not included under the Creative Commons license, users will need to obtain permission from the license holder to reproduce the material. To view a copy of this license, visit http://creativecommons.org/licenses/by/4.0/ 


\section{SCIENTIFIC REP RTS}

\section{Mengcen Wang, Seiji Tachibana, Yuta Murai, Li Li, Sharon Yu Ling Lau, Mengchao Cao, Guonian Zhu, Makoto Hashimoto \& Yasuyuki Hashidoko}

Scientific Reports 6:22596; doi: 10.1038/srep22596; published online 03 March 2016; updated on 20 May 2016

In this Article, the compound ' $(R)-(-)$-2-phenylpropionic acid' was incorrectly given as ' $(R)-(-)$ 2-methylphenylpropionic acid'. In the Results section under subheading 'Interference of the tropolone biosynthetic pathway of $B$. plantarii by PAA analogues',

"Among the PAA analogues tested, $p$-tolylacetic acid, $(R)-(-)-2$-methylphenylpropionic acid, and ( $p$-isopropylphenyl)acetic acid inhibited tropolone production as effectively as IAA at the same concentration (Figure 6)."

should read:

"Among the PAA analogues tested, $p$-tolylacetic acid, '( $R$ )-(-)-2-phenylpropionic acid, and ( $p$-isopropylphenyl) acetic acid inhibited tropolone production as effectively as IAA at the same concentration (Figure 6)."

The correct Figure 6 and its accompanying legend appear below as Figure 1.

Eight compounds, i.e., 3-pyridylacetic acid $\mathrm{HCl}(\mathbf{A})$, 4-hydroxyphenylacetic acid (B), 1-imidazoleacetic acid (C), p-tolylacetic acid (D), (p-isopropylphenyl)acetic acid (E), (R)-(-)-2-phenylpropionic acid (F), $( \pm)$-2-phenylbutyric acid (G), and $(S)-(-)$-3-phenyllactic acid (H), along with indole-3-acetic acid (IAA) (I) as positive control, were tested on $B$. plantarii-impregnated gellan plates, in which Winogradsky's mineral mixture supplemented with $50 \mathrm{~g} \mathrm{~L}^{-1}$ sucrose, $500 \mathrm{mg} \mathrm{L}^{-1}$ yeast extract, and $0.1 \mathrm{mM} \mathrm{Fe}_{2}\left(\mathrm{SO}_{4}\right)_{3}$ was solidified with $10 \mathrm{~g} \mathrm{~L}^{-1}$ gellan gum. An $\mathrm{MeOH}$ solution of each test compound prepared at $0.1,1,10$, and $100 \mathrm{mM}$ was loaded on a paper disk for the assay, and the absolute amount of the test compound on each paper disc is shown in a sub-table in the panel. The sub-number shows the absolute amount of the compound loaded (e.g. A2 is 3-pyridylacetic acid $\cdot \mathrm{HCl}$ at $100 \mathrm{nmol})$. CT is the control ( $\mathrm{MeOH}$ only). Red arrows on the plate photograph indicate tropolone inhibition zone. 
<smiles>O=C(O)Cc1cccnc1</smiles>

3-pyridylacetic acid $\cdot \mathrm{HCl}(\mathbf{A})$<smiles>CC(C)c1ccc(CC(=O)O)cc1</smiles>

(p-isopropylphenyl)acetic acid (E)<smiles>O=C(O)Cc1ccc(O)cc1</smiles><smiles>O=C(O)Cn1ccnc1</smiles>

4-hydroxyphenylacetic acid (B)<smiles>C[C@H](C(=O)O)c1ccccc1</smiles>

(R)-(-)-2-phenylpropionic acid (F)<smiles>CCC(C(=O)O)c1ccccc1</smiles>

$( \pm)$-2-phenylbutyric acid (G)<smiles>Cc1ccc(CC(=O)O)cc1</smiles>

p-tolylacetic acid (D)<smiles>O=C(O)[C@H](O)Cc1ccccc1</smiles>

(S)-(-)-3-phenyllactic acid (H)<smiles>O=C(O)Cc1c[nH]c2ccccc12</smiles>

\begin{tabular}{|c|c|c|c|c|c|c|c|c|c|}
\hline $\begin{array}{c}\text { Compound } \\
\text { Amount loaded }\end{array}$ & A & B & C & D & E & F & G & H & I \\
\hline $\mathbf{1}(1$ umol $)$ & - & - & - & + & ++ & ++ & \pm & - & ++ \\
\hline $\mathbf{2}(100 \mathrm{nmol})$ & - & - & - & - & \pm & - & - & - & - \\
\hline $\mathbf{3}(10 \mathrm{nmol})$ & - & - & - & - & $\mathrm{nt}$ & $\mathrm{nt}$ & - & - & - \\
\hline $\mathbf{4}(1 \mathrm{nmol})$ & - & - & - & - & $\mathrm{nt}$ & $\mathrm{nt}$ & - & - & - \\
\hline
\end{tabular}

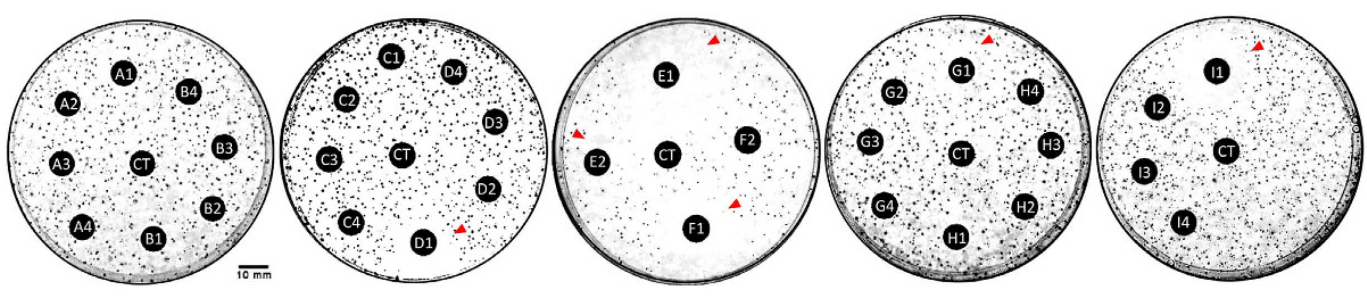

Figure 1.

(c) (i) This work is licensed under a Creative Commons Attribution 4.0 International License. The images or other third party material in this article are included in the article's Creative Commons license, unless indicated otherwise in the credit line; if the material is not included under the Creative Commons license, users will need to obtain permission from the license holder to reproduce the material. To view a copy of this license, visit http://creativecommons.org/licenses/by/4.0/ 